Saudi Journal of Medical and Pharmaceutical Sciences

Abbreviated Key Title: Saudi J Med Pharm Sci

ISSN 2413-4929 (Print) |ISSN 2413-4910 (Online)

Scholars Middle East Publishers, Dubai, United Arab Emirates

Journal homepage: https://saudijournals.com

\title{
Exceptional Acute Epidural Hematoma after a Chronic Subdural Hematoma Evacuation; About One Case
}

\author{
Franck Kouakou $\mathrm{MD}^{1 *}$, V M Kone $\mathrm{MD}^{2}, \mathrm{C}$ Guarneri $\mathrm{MD}^{2}, \mathrm{H}$ Dridi $\mathrm{MD}^{2}$, V Mendes Martins $\mathrm{MD}^{2}$ \\ ${ }^{1}$ Department of neurosurgery, Military Teaching Hospital Mohammed V Rabat; Morocco \\ ${ }^{2}$ Department of neurosurgery, Centre Hospitalier Annecy Genevois; France
}

DOI: $10.36348 /$ simps.2020.v06i12.005

| Received: 09.11.2020 | Accepted: 20.11.2020 | Published: 18.12.2020

*Corresponding author: Franck Kouakou MD

\section{Abstract}

We report a case of evacuation of a chronic subdural hematoma complicated by the occurrence of an epidural hematoma; we discuss the mechanisms that lead to such complication. Eighty-year-old patient admitted in emergency that underwent surgery to evacuate a chronic subdural hematoma. After the evacuation, an epidural hematoma complication was noticed. Several mechanisms have been described by many authors to explain the occurrence of epidural hematomas. The rapid evacuation of a chronic subdural hematoma may be the cause or burr hole that is not performed in the parietal bone can it is mandatory to follow some measures to minimize the risk of developing an epidural hematoma that results in the evacuation of a chronic subdural hematoma.

Keywords: Acute epidural hematoma, chronic subdural hematoma, complication.

Copyright () 2020 The Author(s): This is an open-access article distributed under the terms of the Creative Commons Attribution 4.0 International License (CC BY-NC 4.0) which permits unrestricted use, distribution, and reproduction in any medium for non-commercial use provided the original author and source are credited.

\section{INTRODUCTION}

Chronic subdural hematomas are a common pathology in neurosurgical settings. Although common, many complications can occur following surgical removal of chronic subdural hematomas; some of these complications are very common and others are rare. In this study, we report an exceptional complication caused by a chronic subdural hematoma and discuss its mechanisms of occurrence and management.

\section{Case Report}

It involves an 80-year-old patient with no peculiar past medical history. He was admitted to the emergency room following the progressive occurrence of a deficit in his left hemi-body over a period of two weeks; this deficit was associated with headaches which were inadequately relieved by usual analgesics. He was apyretic, conscious, alert, with good orientation in time and space and had left hemiparesis evaluated at 3/5. A cerebral CT scanner was performed, which allowed us to identify a chronic subdural hematoma in the right fronto-parietal side. This hematoma exerted a mass effect on the midline structure with a brain shift. The bioassay did not identify hematological or biological order abnormalities or blood crass disorder. The activated partial thromboplastin time, and prothrombin time were within normal limits.

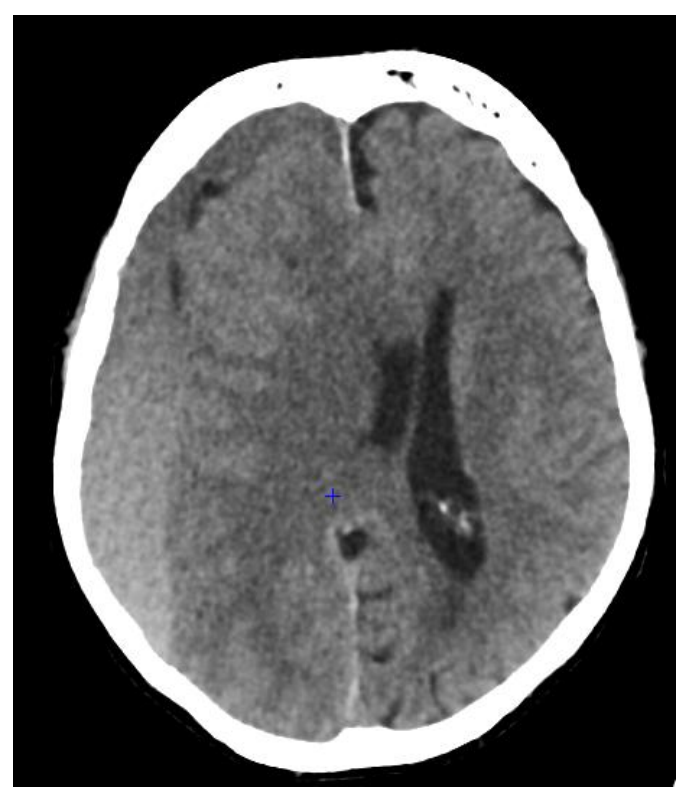

Cerebral CT scan showing the chronic subdural hematoma; before surgery

The patient was admitted to the surgery theater. Under general anesthesia, the hematoma was evacuated through two burr holes: the first was close to the parietal eminence and the second in frontal. The dura opened crosswise and then coagulated on its edges with abundant rinsing until the liquid clears up. A 
Jackson-Pratt drain was placed and connected to a closed system. The vital constant and arterial blood pressures were within normal limits during the surgery. The immediate postoperative results were marked by an improvement in headaches and partial recovery of the motor deficit.

Four hours after surgery, the patient complained of severe headaches without a disorder of consciousness, which motivated the realization of another control brain scan. On the CT scan, we found an extra dural hematoma in front of the frontal hole. The analgesic treatment was optimized and the patient was put on corticosteroid treatment with removal of the drain. Five days after the surgery, his condition remained stable. So, he was discharged and went back home.

At one month, the patient no longer presented headaches and at three months he was able to walk independently and normally.

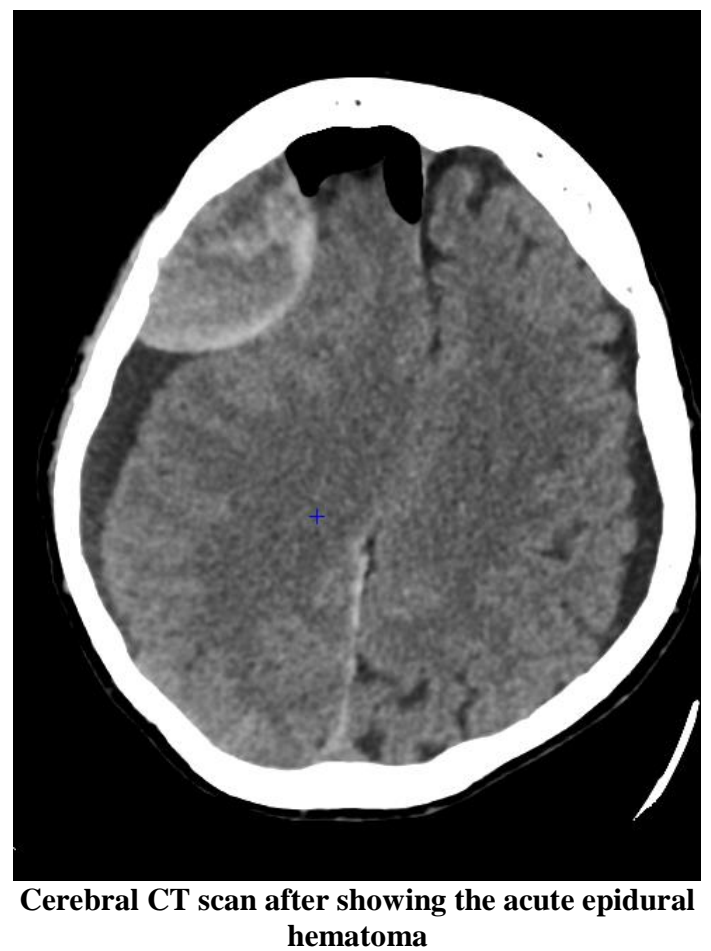

\section{DISCUSSION}

The frequency of occurrence of chronic subdural hematoma is higher in elderly patients; the incidence is 1 to $2 / 100,000$ inhabitants per year [1]. The most accepted pathophysiology is rupture of a cortical vessel following ahead trauma; including minimal head traumas. Blood collection is aggravated by inflammatory phenomena [2]. Different operating techniques are described: membranectomies with craniotomy, evacuation through one or two drill holes, twist drill craniostomy with or without irrigation and with or without drainage, fine percutaneous trepanation and puncture of the dura mater using a needle, etc[2,3].

Although rare, complications due to bleeding giving rise to extra dural hematomas, intra cerebral hematomas, acute subdural hematomas and subarachnoid hemorrhages have been reported several times[4-7]. Factors such as AVMs, anticoagulant treatments, aneurysms and coagulopathies predispose to the onset of these hemorrhagic complications.

Several hypotheses are formulated to explain the occurrence of epidural hematomas. Rapid evacuation of a chronic subdural hematoma results in a sudden drop in ICP that is not quickly compensated [5]. This can be worsened by dehydration during the operation or aspiration of the CSF that is not corrected by intakes. Suddenly withdrawing from sickle cell therapy increases the risk of bleeding. On the other hand, the dura mater nourishing vessels are sparse in the parietal tuber and more abundant in the other parts. The risk of tearing meningeal arteries and provoke an epidural hematoma exists but is lower in the parietal tuber [5]. One of these two hypotheses could explain the occurrence of the epidural hematoma in our case report because the hematoma occurred in the frontal lobe and the evacuation was rapid, inducing a sudden drop in intracranial pressure.

To minimize the risk of the occurrence of epidural hematoma, it is recommended to ensure correct rehydration of the patient, make the trepanation in the parietal tuber [8] and adopt an operating technique that does not promote a sudden drop in intracranial pressure 'Acute'. It is in this context that suggests a fine percutaneous trepanation and the perforation of the dura by a needle. However, despite this technique, YOSHINO and al. reports a case of chronic subdural hematoma occurring during percutaneous trepanation [5]. The hypothesis put forward is the dura mater detachment from the inner surface of the skull. This is the reason why YOSHINO and al. Also advocates that trepanation should not extend beyond the internal table [5].

Any unfavorable development such as worsening of the initial symptoms, headaches or disturbances in consciousness should require urgent control brain CT scanner. If the patient becomes drowsy, an emergency extra dural hematoma evacuation procedure "Acute" should be chosen. If the hematoma is not significant and the patient is conscious, medical treatment may be started with close initial patient monitoring [2].

\section{Conclusion}

It is necessary to think of an epidural hematoma and to urgently carry out a brain CT scan in any patient during or after the procedure to evacuate 
subdural hematoma. The clinical manifestations can occur suddenly during the operation or in the immediate aftermath.

The best way to manage such occurrences will depend on the clinical condition of the patient and the size of the hematoma.

\section{REFERENCES}

1. Drapkin, A. J. (1991). Chronic subdural hematoma: pathophysiological basis for treatment. British journal of neurosurgery, 5(5), 467-473.

2. Akpinar, A., Ucler, N., Erdogan, U., \& Yucetas, C. S. (2015). Epidural hematoma complication after rapid chronic subdural hematoma evacuation: A case report. The American journal of case reports, 16, 430.

3. Lee, J.K. (2000). Small Craniotomy vs. Burr-Hole Craniostomy for Chronic Subdural Hematoma; $J$ Korean Neurosurg Soc, 46 : 210-214

4. Mori, K., \& Maeda, M. (2001). Surgical treatment of chronic subdural hematoma in 500 consecutive cases: clinical characteristics, surgical outcome, complications, and recurrence rate. Neurologia medico-chirurgica, 41(8), 371-381.

5. Yoshino, Y., Aoki, N., Oikawa, A., \& Ohno, K. (2000). Acute epidural hematoma developing during twist-drill craniostomy: a complication of percutaneous subdural tapping for the treatment of chronic subdural hematoma. Surgical neurology, 53(6), 601-604.

6. Akhaddar, A., Ajja, A., Elmostarchid, B., \& Boucetta, M. (2008). Combined epidural and intracerebral hematomas after evacuation of bilateral chronic subdural hematoma. Neurochirurgie, 54(6), 728-730.

7. N'Dri Oka, D., Soro, L., Broalet, E., Varlet, G., \& Ba Zeze, V. (2000). L'hématome extradural, une complication possible de la chirurgie de l'hématome sousdural chronique. Neurochirurgie, 46, 575.

8. Sucu, H. K., Gökmen, M., Ergin, A., Bezircioğlu, H., \& Gökmen, A. (2007). Is there a way to avoid surgical complications of twist drill craniostomy for evacuation of a chronic subdural hematoma?. Acta neurochirurgica, 149(6), $597-$ 599. 